\title{
Nutritional composition of commercially-produced $100 \%$ orange juice reveals large variability in vascular health bioactive, hesperidin
}

\section{Abstract}

It is well-accepted that fruits contain a wide array of nutrients and bioactive substances which may contribute towards the prevention of chronic disease. Citrus fruits, as well as their juices, are recognised for their rich polyphenol content, specifically the flavanones; hesperidin, naringin and narirutin. Citrus fruits and juices are also rich in vitamin $\mathrm{C}$ and provide a source of folate, potassium and provitamin A.

Randomised controlled trials have reported significant improvements to vascular function and blood pressure following consumption of $100 \%$ orange juice $(\mathrm{OJ})$ with the effects potentially mediated through hesperidin and/or potassium (which has an EU claim for supporting normal blood pressure). Further clinical research may be facilitated by up-to-date information on the composition of OJ.

By auditing more than 350 fruit processing companies worldwide, the non-profit organisation SGF International collects authentic OJ samples for regular compliance testing. Samples of unpasteurized OJ from the production line are removed by trained staff immediately post-extraction and frozen at $-18^{\circ} \mathrm{C}$ within a few minutes. The samples are posted in this state to accredited laboratories where key nutrients and bioactives are tested. SGF's Database of Authentic Samples (2018) provides the following data (mg/litre) as sample size, mean, standard deviation, minimum, maximum for hesperidin (231, 520, 175, 109, 1160), L-ascorbic acid (615, 450, 98, 120, $721)$, potassium $(1242,1758,204,1197,2340)$, total carotenoids $(575,7,3,2,21)$ and pectins $(1043,334,132,19,932)$.

On average, there was more hesperidin in OJ than vitamin C, giving an estimated composition of $78 \mathrm{mg}$ hesperidin and $67.5 \mathrm{mg}$ vitamin C per $150 \mathrm{~mL}$ serving. Samples from Chinese producers had the greatest variability in hesperidin (109-1160 mg/L) while samples from Argentina, South Africa and Spain had the smallest variability. When OJ from not-from-concentrate $(\mathrm{n}=62)$ and concentrate $(n=169)$ were compared, mean levels of hesperidin were significantly higher in the not-from-concentrate juice $(576 \mathrm{mg} / \mathrm{L}$ [sd 228 $\mathrm{mg} / \mathrm{L}$ ] vs. $500 \mathrm{mg} / \mathrm{L}$ [sd $146 \mathrm{mg} / \mathrm{L}$ ]; $\mathrm{P}=0.016$; Welch's t-test).

Vascular benefits in humans have been detected at OJ intakes of $500 \mathrm{ml} /$ day or hesperidin intakes of $290 \mathrm{mg} / \mathrm{day}$ (Morand et al. 2011). From a quality assurance perspective, the AIJN sets an acceptable range for hesperidin as 250-700mg/L. Raising the upper cut-off could increase the hesperidin content of commercially-available OJ with potential benefits for vascular health.

Morand C et al. (2011) Am J Clin Nutr 93: 73-80.

\section{Conflict of Interest}

Prof. De Rycker and Dr Ruxton are consultants for AIJN, the European Fruit Juice Association. Prof. Schweiggert serves on the AIJN Scientific Expert Panel 